\title{
Generalizations of Steffensen's inequality via two-point Abel-Gontscharoff polynomial
}

\author{
Josip Pečarić, Anamarija Perušić Pribanić and Ksenija Smoljak \\ Kalamir
}

\begin{abstract}
Using two-point Abel-Gontscharoff interpolating polynomial some new generalizations of Steffensen's inequality for $n$-convex functions are obtained and some Ostrowski-type inequalities related to obtained generalizations are given. Furthermore, using the Čebyšev functional some new bounds for the remainder in obtained generalizations are proven and related Grüss-type inequalities are given.
\end{abstract}

\section{Introduction}

The two-Point Abel-Gontscharoff interpolation problem is a particular case of Abel-Gontscharoff interpolation problem introduced in 1935 by Whittaker [11] and subseququently by Gontscharoff [4] and Davis [3]. In [1] this interpolation problem is also reffered to as the two-point right focal interpolation problem. Let $f \in C^{n}[a, b](n \geq 2)$ and let $P_{A G 2}$ be its two-point Abel-Gontscharoff interpolating polynomial then

$$
f(t)=P_{A G 2}(t)+e_{A G 2}(t)
$$

Key Words: Steffensen's inequality, generalizations, $n$-convex functions, Abel-Gontscharoff polynomial, Ostrowski-type inequality, Grüss-type inequality.

2010 Mathematics Subject Classification: Primary 26D15; Secondary 26A51. 
where $P_{A G 2}$ is the polynomial of degree $n-1$ defined by

$$
\begin{aligned}
P_{A G 2}(t)=\sum_{i=0}^{\alpha} & \frac{\left(t-a_{1}\right)^{i}}{i !} f^{(i)}\left(a_{1}\right) \\
& +\sum_{j=0}^{n-\alpha-2}\left[\sum_{i=0}^{j} \frac{\left(t-a_{1}\right)^{\alpha+1+i}\left(a_{1}-a_{2}\right)^{j-i}}{(\alpha+1+i) !(j-i) !}\right] f^{(\alpha+1+j)}\left(a_{2}\right)
\end{aligned}
$$

and the associated error can be expressed by

$$
e_{A G 2}(t)=\int_{a}^{b} g_{A G 2}(t, s) f^{(n)}(s) d s .
$$

The corresponding Green function $g_{A G 2}(t, s)$ from (1.2) is defined by

$$
g_{A G 2}(t, s)=\frac{1}{(n-1) !} \begin{cases}\sum_{i=0}^{\alpha}\left(\begin{array}{c}
n-1 \\
i
\end{array}\right)\left(t-a_{1}\right)^{i}\left(a_{1}-s\right)^{n-i-1}, & a \leq s \leq t, \\
-\sum_{i=\alpha+1}^{n-1}\left(\begin{array}{c}
n-1 \\
i
\end{array}\right)\left(t-a_{1}\right)^{i}\left(a_{1}-s\right)^{n-i-1}, & t \leq s \leq b .\end{cases}
$$

The polynomial $P_{A G 2}$ satisfies the following conditions called the two-point right focal conditions ([1, p. 172])

$$
\begin{aligned}
& P_{A G 2}^{(i)}\left(a_{1}\right)=f^{(i)}\left(a_{1}\right), \quad 0 \leq i \leq \alpha \\
& P_{A G 2}^{(i)}\left(a_{2}\right)=f^{(i)}\left(a_{2}\right), \quad \alpha+1 \leq i \leq n-1, a \leq a_{1}<a_{2} \leq b .
\end{aligned}
$$

These conditions are a particular case of the general Abel-Gontscharoff interpolation conditions

$$
P_{A G}^{(i)}\left(a_{i+1}\right)=f^{(i)}\left(a_{i+1}\right), \quad 0 \leq i \leq n-1, a \leq a_{1} \leq a_{2} \leq \cdots \leq a_{n} \leq b
$$

Some generalizations of Steffensen's inequality via Abel-Gontscharoff polynomial using the difference of integrals on two intervals were obtained in [7]. The aim of this paper is to obtain some new generalizations of Steffensen's inequality via two-point Abel-Gontscharoff polynomial using different reasoning from the one used in [7]. This inequality was first given and proved by Steffensen in 1918 in paper [10]. A comprehensive survey on Steffensen's inequality, its generalizations and applications can be found in [9].

The well-known Steffensen inequality states: 
Theorem 1.1. Suppose that $f$ is nonincreasing and $g$ is integrable on $[a, b]$ with $0 \leq g \leq 1$ and $\lambda=\int_{a}^{b} g(t) d t$. Then we have

$$
\int_{b-\lambda}^{b} f(t) d t \leq \int_{a}^{b} f(t) g(t) d t \leq \int_{a}^{a+\lambda} f(t) d t .
$$

The inequalities are reversed for $f$ nondecreasing.

In [5] Jakšetić and Pečarić generalized Steffensen's inequality for positive measures using identites

$$
\begin{aligned}
\int_{[a, a+\lambda]} f(t) d \mu(t) & -\int_{[a, b]} f(t) g(t) d \mu(t) \\
& =\int_{[a, a+\lambda]} f(t)(1-g(t)) d \mu(t)-\int_{(a+\lambda, b]} f(t) g(t) d \mu(t)
\end{aligned}
$$

and

$$
\begin{aligned}
\int_{[a, b]} f(t) g(t) d \mu(t) & -\int_{(b-\lambda, b]} f(t) d \mu(t) \\
& =\int_{[a, b-\lambda]} f(t) g(t) d \mu(t)-\int_{(b-\lambda, b]} f(t)(1-g(t)) d \mu(t) .
\end{aligned}
$$

Mitrinović stated in [6] that the inequalities in (1.3) follow from the above identities for $d \mu(t)=d t$.

The above identities for $d \mu(t)=p(t) d t$ will be the starting point for our generalizations of Steffensen's inequality.

In this paper we use the two-point Abel-Gontscharoff polynomial to obtain some new identities related to Steffensen's inequality. Using our new identities we generalize Steffensen's inequality for $n$-convex functions. In Section 3 we give the Ostrowski-type inequalities related to obtained generalizations. We conclude this paper with some new bounds for the remainder in obtained identities using inequalities for the Čebyšev functional and with some Grüsstype inequalities.

Throughout the paper, it is assumed that all integrals under consideration exist and that they are finite.

\section{Generalizations of Steffensen's inequality}

In this section we obtain generalizations of Steffensen's inequality for $n$-convex functons using identity (1.1). 
Theorem 2.1. Let $f \in C^{n}[a, b]$ for $n \geq 3$ and let $g, p:[a, b] \rightarrow \mathbb{R}$ be integrable functions such that $p$ is positive and $0 \leq g \leq 1$. Let $\int_{a}^{a+\lambda} p(t) d t=\int_{a}^{b} g(t) p(t) d t$ and let the function $G_{1}$ be defined by

$$
G_{1}(x)= \begin{cases}\int_{a}^{x}(1-g(t)) p(t) d t, & x \in[a, a+\lambda], \\ \int_{x}^{b} g(t) p(t) d t, & x \in[a+\lambda, b] .\end{cases}
$$

Then

$$
\begin{aligned}
& \int_{a}^{a+\lambda} f(t) p(t) d t-\int_{a}^{b} f(t) g(t) p(t) d t+\sum_{i=0}^{\alpha} \frac{f^{(i+1)}(a)}{i !} \int_{a}^{b} G_{1}(x)(x-a)^{i} d x \\
& +\sum_{j=0}^{n-\alpha-3} f^{(\alpha+j+2)}(a+\lambda)\left[\sum_{i=0}^{j} \frac{(-\lambda)^{j-i}}{(\alpha+1+i) !(j-i) !}\left(\int_{a}^{b}(x-a)^{\alpha+1+i} G_{1}(x) d x\right)\right] \\
& =-\int_{a}^{b}\left(\int_{a}^{b} G_{1}(x) g_{A G 2}(x, s) d x\right) f^{(n)}(s) d s .
\end{aligned}
$$

Proof. Using identity (1.4) for $d \mu(t)=p(t) d t$ and integration by parts we have

$$
\begin{aligned}
& \int_{a}^{a+\lambda} f(t) p(t) d t-\int_{a}^{b} f(t) g(t) p(t) d t \\
& =\int_{a}^{a+\lambda}[f(t)-f(a+\lambda)][1-g(t)] p(t) d t+\int_{a+\lambda}^{b}[f(a+\lambda)-f(t)] g(t) p(t) d t \\
& =-\int_{a}^{a+\lambda}\left[\int_{a}^{x}(1-g(t)) p(t) d t\right] d f(x)-\int_{a+\lambda}^{b}\left[\int_{x}^{b} g(t) p(t) d t\right] d f(x) \\
& =-\int_{a}^{b} G_{1}(x) d f(x)=-\int_{a}^{b} G_{1}(x) f^{\prime}(x) d x .
\end{aligned}
$$

Applying identity (1.1) to $f^{\prime}$, taking $a_{1}=a, a_{2}=a+\lambda$ and replacing $n$ with $n-1$ we have

$$
\begin{aligned}
f^{\prime}(x) & =\sum_{i=0}^{\alpha} \frac{f^{(i+1)}(a)}{i !}(x-a)^{i}+\sum_{j=0}^{n-\alpha-3} f^{(\alpha+j+2)}(a+\lambda)\left[\sum_{i=0}^{j} \frac{(-\lambda)^{j-i}(x-a)^{\alpha+1+i}}{(\alpha+1+i) !(j-i) !}\right] \\
& +\int_{a}^{b} g_{A G 2}(x, s) f^{(n)}(s) d s .
\end{aligned}
$$


Now using (2.3) we obtain

$$
\begin{aligned}
& \int_{a}^{b} G_{1}(x) f^{\prime}(x) d x=\sum_{i=0}^{\alpha} \frac{f^{(i+1)}(a)}{i !} \int_{a}^{b} G_{1}(x)(x-a)^{i} d x \\
& +\sum_{j=0}^{n-\alpha-3} f^{(\alpha+j+2)}(a+\lambda)\left[\sum_{i=0}^{j} \frac{(-\lambda)^{j-i}}{(\alpha+1+i) !(j-i) !}\left(\int_{a}^{b}(x-a)^{\alpha+1+i} G_{1}(x) d x\right)\right] \\
& +\int_{a}^{b} G_{1}(x)\left(\int_{a}^{b} g_{A G 2}(x, s) f^{(n)}(s) d s\right) d x
\end{aligned}
$$

After applying Fubini's theorem on the last term in (2.4) we obtain (2.2).

Theorem 2.2. Let $f \in C^{n}[a, b]$ for $n \geq 3$ and let $g, p:[a, b] \rightarrow \mathbb{R}$ be integrable functions such that $p$ is positive and $0 \leq g \leq 1$. Let $\int_{b-\lambda}^{b} p(t) d t=\int_{a}^{b} g(t) p(t) d t$ and let the function $G_{2}$ be defined by

$$
G_{2}(x)= \begin{cases}\int_{a}^{x} g(t) p(t) d t, & x \in[a, b-\lambda], \\ \int_{x}^{b}(1-g(t)) p(t) d t, & x \in[b-\lambda, b] .\end{cases}
$$

Then

$$
\begin{aligned}
& \int_{a}^{b} f(t) g(t) p(t) d t-\int_{b-\lambda}^{b} f(t) p(t) d t+\sum_{i=0}^{\alpha} \frac{f^{(i+1)}(b-\lambda)}{i !} \int_{a}^{b} G_{2}(x)(x-b+\lambda)^{i} d x \\
& +\sum_{j=0}^{n-\alpha-3} f^{(\alpha+j+2)}(b)\left(\sum_{i=0}^{j} \frac{(-\lambda)^{j-i}}{(\alpha+1+i) !(j-i) !}\left(\int_{a}^{b}(x-b+\lambda)^{\alpha+1+i} G_{2}(x) d x\right)\right) \\
& =-\int_{a}^{b}\left(\int_{a}^{b} G_{2}(x) g_{A G 2}(x, s) d x\right) f^{(n)}(s) d s .
\end{aligned}
$$

Proof. Similar to the proof of Theorem 2.1 using identity (1.5) for $d \mu(t)=$ $p(t) d t$.

Now, using the above obtained identites we give generalization of Steffensen's inequality for $n$-convex functions.

Theorem 2.3. Let $f \in C^{n}[a, b]$ for $n \geq 3$ and let $g, p:[a, b] \rightarrow \mathbb{R}$ be integrable functions such that $p$ is positive and $0 \leq g \leq 1$. Let $\int_{a}^{a+\lambda} p(t) d t=\int_{a}^{b} g(t) p(t) d t$ and let the function $G_{1}$ be defined by (2.1). If $f$ is $n$-convex and

$$
\int_{a}^{b} G_{1}(x) g_{A G 2}(x, s) d x \leq 0, \quad s \in[a, b],
$$


then

$$
\begin{aligned}
& \int_{a}^{b} f(t) g(t) p(t) d t \leq \int_{a}^{a+\lambda} f(t) p(t) d t+\sum_{i=0}^{\alpha} \frac{f^{(i+1)}(a)}{i !} \int_{a}^{b} G_{1}(x)(x-a)^{i} d x \\
& +\sum_{j=0}^{n-\alpha-3} f^{(\alpha+j+2)}(a+\lambda)\left[\sum_{i=0}^{j} \frac{(-\lambda)^{j-i}}{(\alpha+1+i) !(j-i) !}\left(\int_{a}^{b}(x-a)^{\alpha+1+i} G_{1}(x) d x\right)\right] .
\end{aligned}
$$

Proof. If the function $f$ is $n$-convex, without loss of generality we can assume that $f$ is $n$-times differentiable and $f^{(n)} \geq 0$ see [8, p. 16 and p. 293]. Now we can apply Theorem 2.1 to obtain (2.8).

Theorem 2.4. Let $f \in C^{n}[a, b]$ for $n \geq 3$ and let $g, p:[a, b] \rightarrow \mathbb{R}$ be integrable functions such that $p$ is positive and $0 \leq g \leq 1$. Let $\int_{b-\lambda}^{b} p(t) d t=\int_{a}^{b} g(t) p(t) d t$ and let the function $G_{2}$ be defined by (2.5). If $f$ is $n$-convex and

$$
\int_{a}^{b} G_{2}(x) g_{A G 2}(x, s) d x \leq 0, \quad s \in[a, b],
$$

then

$$
\begin{aligned}
& \int_{a}^{b} f(t) g(t) p(t) d t \geq \int_{b-\lambda}^{b} f(t) p(t) d t-\sum_{i=0}^{\alpha} \frac{f^{(i+1)}(b-\lambda)}{i !} \int_{a}^{b} G_{2}(x)(x-b+\lambda)^{i} d x \\
& -\sum_{j=0}^{n-\alpha-3} f^{(\alpha+j+2)}(b)\left(\sum_{i=0}^{j} \frac{(-\lambda)^{j-i}}{(\alpha+1+i) !(j-i) !}\left(\int_{a}^{b}(x-b+\lambda)^{\alpha+1+i} G_{2}(x) d x\right)\right)
\end{aligned}
$$

Proof. Similar to the proof of Theorem 2.3.

Remark 2.1. If the integrals in (2.7) and (2.9) are nonnegative, then the reverse inequalities in (2.8) and (2.10) hold.

Taking $p \equiv 1$ and $n=3$ in previous theorems we obtain the following corollary.

Corollary 2.1. Let $f \in C^{3}[a, b]$ and let $g:[a, b] \rightarrow \mathbb{R}$ be an integrable function such that $0 \leq g \leq 1$. Let $\lambda=\int_{a}^{b} g(t) d t$.

(i) If $f$ is 3-convex and

$$
\begin{aligned}
& -\frac{1}{3} \int_{a}^{s} x^{3} g(x) d x+s \int_{a}^{s} x^{2} g(x) d x+s^{2} \int_{s}^{b} x g(x) d x+\left(a^{2}-2 a s\right) \int_{a}^{b} x g(x) d x \\
& +\frac{s^{3}}{3} \int_{a}^{s} g(x) d x \leq \frac{(s-a)^{4}}{12}-\frac{(s-a)^{2}}{2}\left(\lambda a-\frac{\lambda^{2}}{2}\right), \quad s \in[a, a+\lambda],
\end{aligned}
$$




$$
\begin{aligned}
& -\frac{1}{3} \int_{a}^{s} x^{3} g(x) d x+s \int_{a}^{s} x^{2} g(x) d x+s^{2} \int_{s}^{b} x g(x) d x+\left(a^{2}-2 a s\right) \int_{a}^{b} x g(x) d x \\
& -\frac{s^{3}}{3} \int_{s}^{b} g(x) d x \leq\left(\frac{2 a^{3}}{3}-s a^{2}\right) \lambda+\frac{(s-a) \lambda^{3}}{3}-\frac{\lambda^{4}}{12}, \quad s \in[a+\lambda, b],
\end{aligned}
$$

then

$$
\begin{aligned}
\int_{a}^{b} f(t) g(t) d t & \leq \int_{a}^{a+\lambda} f(t) d t+f^{\prime}(a)\left(\int_{a}^{b} x g(x) d x-a \lambda-\frac{\lambda^{2}}{2}\right) \\
& +\frac{f^{\prime \prime}(a+\lambda)}{2}\left(\int_{a}^{b} g(x)(x-a)^{2} d x-\frac{\lambda^{3}}{3}\right) .
\end{aligned}
$$

(ii) If $f$ is 3-convex and

$$
\begin{aligned}
& \frac{1}{3} \int_{a}^{s} x^{3} g(x) d x-s \int_{a}^{s} x^{2} g(x) d x-\left((b-\lambda)^{2}-2 s(b-\lambda)\right) \int_{a}^{b} x g(x) d x \\
& -s^{2} \int_{s}^{b} x g(x) d x-\frac{s^{3}}{3} \int_{a}^{s} g(x) d x \leq \frac{(b-\lambda-s)^{2}}{2}\left(\frac{\lambda^{2}}{2}-\lambda b\right), \quad s \in[a, b-\lambda], \\
& \frac{1}{3} \int_{a}^{s} x^{3} g(x) d x-s \int_{a}^{s} x^{2} g(x) d x-\left[(b-\lambda)^{2}-2 s(b-\lambda)\right] \int_{a}^{b} x g(x) d x \\
& -s^{2} \int_{s}^{b} x g(x) d x+\frac{s^{3}}{3} \int_{s}^{b} g(x) d x \leq-\frac{(s-b+\lambda)^{4}}{12} \\
& \left.-\frac{(s-b+\lambda)^{3} \lambda}{2}\left[\frac{\lambda}{3}+\frac{2}{3}(b-s)\right]-\lambda(b-\lambda)^{2}\left[\frac{2}{3}(b-\lambda)-s\right)\right], \quad s \in[b-\lambda, b],
\end{aligned}
$$

$$
\begin{aligned}
\int_{a}^{b} f(t) g(t) d t & \geq \int_{b-\lambda}^{b} f(t) d t+f^{\prime}(b-\lambda)\left(\frac{\lambda^{2}}{2}-b \lambda+\int_{a}^{b} x g(x) d x\right) \\
& -\frac{f^{\prime \prime}(b)}{2}\left(\frac{\lambda^{3}}{3}-\int_{a}^{b} g(x)(x-b+\lambda)^{2} d x\right) .
\end{aligned}
$$

\section{Ostrowski-type inequalities}

In this section we give the Ostrowski-type inequalities related to generalizations obtained in the previous section. 
Theorem 3.1. Suppose that all assumptions of Theorem 2.1 hold. Assume $(p, q)$ is a pair of conjugate exponents, that is $1 \leq p, q \leq \infty, 1 / p+1 / q=1$. Let $\left|f^{(n)}\right|^{p}:[a, b] \rightarrow \mathbb{R}$ be an $R$-integrable function for some $n \geq 3$. Then we have

$$
\begin{aligned}
& \mid \int_{a}^{a+\lambda} f(t) p(t) d t-\int_{a}^{b} f(t) g(t) p(t) d t+\sum_{i=0}^{\alpha} \frac{f^{(i+1)}(a)}{i !} \int_{a}^{b} G_{1}(x)(x-a)^{i} d x \\
& +\sum_{j=0}^{n-\alpha-3} f^{(\alpha+j+2)}(a+\lambda)\left[\sum_{i=0}^{j} \frac{(-\lambda)^{j-i}}{(\alpha+1+i) !(j-i) !}\left(\int_{a}^{b}(x-a)^{\alpha+1+i} G_{1}(x) d x\right)\right] \mid \\
& \leq\left\|f^{(n)}\right\|_{p}\left(\int_{a}^{b}\left|\int_{a}^{b} G_{1}(x) g_{A G 2}(x, s) d x\right|^{q} d s\right)^{\frac{1}{q}} .
\end{aligned}
$$

The constant on the right-hand side of (3.1) is sharp for $1<p \leq \infty$ and the best possible for $p=1$.

Proof. Let's denote

$$
C(s)=\int_{a}^{b} G_{1}(x) g_{A G 2}(x, s) d x .
$$

By taking the modulus of (2.2) and applying Hölder's inequality we obtain

$$
\begin{aligned}
& \mid \int_{a}^{a+\lambda} f(t) p(t) d t-\int_{a}^{b} f(t) g(t) p(t) d t+\sum_{i=0}^{\alpha} \frac{f^{(i+1)}(a)}{i !} \int_{a}^{b} G_{1}(x)(x-a)^{i} d x \\
& +\sum_{j=0}^{n-\alpha-3} f^{(\alpha+j+2)}(a+\lambda)\left[\sum_{i=0}^{j} \frac{(-\lambda)^{j-i}}{(\alpha+1+i) !(j-i) !}\left(\int_{a}^{b}(x-a)^{\alpha+1+i} G_{1}(x) d x\right)\right] \mid \\
& =\left|\int_{a}^{b} C(s) f^{(n)}(s) d s\right| \leq\left\|f^{(n)}\right\|\left(\int_{a}^{b}|C(s)|^{q} d s\right)^{\frac{1}{q}} .
\end{aligned}
$$

For the proof of the sharpness of the constant $\left(\int_{a}^{b}|C(s)|^{q} d s\right)^{\frac{1}{q}}$ let us find a function $f$ for which the equality in (3.1) is obtained.

For $1<p<\infty$ take $f$ to be such that

$$
f^{(n)}(s)=\operatorname{sgn} C(s)|C(s)|^{\frac{1}{p-1}} .
$$


For $p=\infty$ take $f^{(n)}(s)=\operatorname{sgn} C(s)$.

For $p=1$ we prove that

$$
\left|\int_{a}^{b} C(s) f^{(n)}(s) d s\right| \leq \max _{s \in[a, b]}|C(s)|\left(\int_{a}^{b}\left|f^{(n)}(s)\right| d s\right)
$$

is the best possible inequality. Suppose that $|C(s)|$ attains its maximum at $s_{0} \in[a, b]$. First we assume that $C\left(s_{0}\right)>0$. For $\varepsilon$ small enough we define $f_{\varepsilon}(s)$ by

$$
f_{\varepsilon}(s)= \begin{cases}0, & a \leq s \leq s_{0}, \\ \frac{1}{\varepsilon n !}\left(s-s_{0}\right)^{n}, & s_{0} \leq s \leq s_{0}+\varepsilon, \\ \frac{1}{n !}\left(s-s_{0}\right)^{n-1}, & s_{0}+\varepsilon \leq s \leq b .\end{cases}
$$

Then for $\varepsilon$ small enough

$$
\left|\int_{a}^{b} C(s) f^{(n)}(s) d s\right|=\left|\int_{s_{0}}^{s_{0}+\varepsilon} C(s) \frac{1}{\varepsilon} d s\right|=\frac{1}{\varepsilon} \int_{s_{0}}^{s_{0}+\varepsilon} C(s) d s .
$$

Now from the inequality (3.2) we have

$$
\frac{1}{\varepsilon} \int_{s_{0}}^{s_{0}+\varepsilon} C(s) d s \leq C\left(s_{0}\right) \int_{s_{0}}^{s_{0}+\varepsilon} \frac{1}{\varepsilon} d s=C\left(s_{0}\right) .
$$

Since,

$$
\lim _{\varepsilon \rightarrow 0} \frac{1}{\varepsilon} \int_{s_{0}}^{s_{0}+\varepsilon} C(s) d s=C\left(s_{0}\right)
$$

the statement follows. In the case $C\left(s_{0}\right)<0$, we define $f_{\varepsilon}(s)$ by

$$
f_{\varepsilon}(s)= \begin{cases}\frac{1}{n !}\left(s-s_{0}-\varepsilon\right)^{n-1},, & a \leq s \leq s_{0} \\ -\frac{1}{\varepsilon n !}\left(s-s_{0}-\varepsilon\right)^{n}, & s_{0} \leq s \leq s_{0}+\varepsilon \\ 0, & s_{0}+\varepsilon \leq s \leq b\end{cases}
$$

and the rest of the proof is the same as above.

Using identity (2.6) we obtain the following result.

Theorem 3.2. Suppose that all assumptions of Theorem 2.2 hold. Assume $(p, q)$ is a pair of conjugate exponents, that is $1 \leq p, q \leq \infty, 1 / p+1 / q=1$. Let $\left|f^{(n)}\right|^{p}:[a, b] \rightarrow \mathbb{R}$ be an R-integrable function for some $n \geq 3$. Then we 
have

$$
\begin{aligned}
& \mid \int_{a}^{b} f(t) g(t) p(t) d t-\int_{b-\lambda}^{b} f(t) p(t) d t+\sum_{i=0}^{\alpha} \frac{f^{(i+1)}(b-\lambda)}{i !} \int_{a}^{b} G_{2}(x)(x-b+\lambda)^{i} d x \\
& +\sum_{j=0}^{n-\alpha-3} f^{(\alpha+j+2)}(b)\left(\sum_{i=0}^{j} \frac{(-\lambda)^{j-i}}{(\alpha+1+i) !(j-i) !}\left(\int_{a}^{b}(x-b+\lambda)^{\alpha+1+i} G_{2}(x) d x\right)\right) \mid \\
& \leq\left\|f^{(n)}\right\|_{p}\left(\int_{a}^{b}\left|\int_{a}^{b} G_{2}(x) g_{A G 2}(x, s) d x\right|^{q} d s\right)^{\frac{1}{q}} .
\end{aligned}
$$

The constant on the right-hand side of (3.3) is sharp for $1<p \leq \infty$ and the best possible for $p=1$.

Proof. Similar to the proof of Theorem 3.1.

Taking $p \equiv 1$ and $n=3$ in Theorems 3.1 and 3.2 we obtain the following corollaries.

Corollary 3.1. Let $f:[a, b] \rightarrow \mathbb{R}$ be such that $f \in C^{3}[a, b]$, let $g:[a, b] \rightarrow \mathbb{R}$ be an integrable function such that $0 \leq g \leq 1$ and let $\lambda=\int_{a}^{b} g(t) d t$. Assume $(p, q)$ is a pair of conjugate exponents, that is $1 \leq p, q \leq \infty, 1 / p+1 / q=1$. Let $\left|f^{\prime \prime \prime}\right|^{p}:[a, b] \rightarrow \mathbb{R}$ be an R-integrable function. Then for $1<p \leq \infty$ we have

$$
\begin{aligned}
& \mid \int_{a}^{a+\lambda} f(t) d t-\int_{a}^{b} f(t) g(t) d t+f^{\prime}(a)\left(\int_{a}^{b} x g(x) d x-a \lambda-\frac{\lambda^{2}}{2}\right) \\
& +\frac{f^{\prime \prime}(a+\lambda)}{2}\left(\int_{a}^{b} g(x)(x-a)^{2} d x-\frac{\lambda^{3}}{3}\right) \mid \leq\left\|f^{\prime \prime \prime}\right\|_{p}\left(\int_{a}^{a+\lambda} \mid-\frac{1}{3} \int_{a}^{s} x^{3} g(x) d x\right. \\
& +s \int_{a}^{s} x^{2} g(x) d x+s^{2} \int_{s}^{b} x g(x) d x+\left(a^{2}-2 a s\right) \int_{a}^{b} x g(x) d x+\frac{s^{3}}{3} \int_{a}^{s} g(x) d x-\frac{(s-a)^{4}}{12} \\
& +\left.\frac{(s-a)^{2}}{2}\left(\lambda a-\frac{\lambda^{2}}{2}\right)\right|^{q} d t+\int_{a+\lambda}^{b} \mid-\frac{1}{3} \int_{a}^{s} x^{3} g(x) d x+s \int_{a}^{s} x^{2} g(x) d x+s^{2} \int_{s}^{b} x g(x) d x \\
& \left.+\left(a^{2}-2 a s\right) \int_{a}^{b} x g(x) d x-\frac{s^{3}}{3} \int_{s}^{b} g(x) d x-\left(\frac{2 a^{3}}{3}-s a^{2}\right) \lambda-\frac{(s-a) \lambda^{3}}{3}+\left.\frac{\lambda^{4}}{12}\right|^{q} d t\right)^{\frac{1}{q}} .
\end{aligned}
$$

and the constant on the right-hand side of (3.4) is sharp, while for $p=1$ we 
have

$$
\begin{aligned}
& \mid \int_{a}^{a+\lambda} f(t) d t-\int_{a}^{b} f(t) g(t) d t+f^{\prime}(a)\left(\int_{a}^{b} x g(x) d x-a \lambda-\frac{\lambda^{2}}{2}\right) \\
& +\frac{f^{\prime \prime}(a+\lambda)}{2}\left(\int_{a}^{b} g(x)(x-a)^{2} d x-\frac{\lambda^{3}}{3}\right) \mid \leq\left\|f^{\prime \prime \prime}\right\|_{1} \max \left\{M_{1}, M_{2}\right\}
\end{aligned}
$$

where

$$
\begin{aligned}
& M_{1}=\max _{s \in[a, a+\lambda]}\{-\frac{1}{3} \int_{a}^{s} x^{3} g(x) d x+s \int_{a}^{s} x^{2} g(x) d x+s^{2} \int_{s}^{b} x g(x) d x \\
&+\left(a^{2}-2 a s\right) \int_{a}^{b} x g(x) d x+\frac{s^{3}}{3} \int_{a}^{s} g(x) d x-\frac{(s-a)^{4}}{12} \\
&\left.+\frac{(s-a)^{2}}{2}\left(\lambda a-\frac{\lambda^{2}}{2}\right)\right\}, \\
& M_{2}=\max _{s \in[a+\lambda, b]}\left\{-\frac{1}{3} \int_{a}^{s} x^{3} g(x) d x+s \int_{a}^{s} x^{2} g(x) d x+s^{2} \int_{s}^{b} x g(x) d x\right. \\
&+\left(a^{2}-2 a s\right) \int_{a}^{b} x g(x) d x-\frac{s^{3}}{3} \int_{s}^{b} g(x) d x-\left(\frac{2 a^{3}}{3}-s a^{2}\right) \lambda \\
&\left.-\frac{(s-a) \lambda^{3}}{3}+\frac{\lambda^{4}}{12}\right\} .
\end{aligned}
$$

and the constant on the right-hand side of (3.5) is the best possible.

Corollary 3.2. Let $f:[a, b] \rightarrow \mathbb{R}$ be such that $f \in C^{3}[a, b]$, let $g:[a, b] \rightarrow \mathbb{R}$ be an integrable function such that $0 \leq g \leq 1$ and let $\lambda=\int_{a}^{b} g(t) d t$. Assume $(p, q)$ is a pair of conjugate exponents, that is $1 \leq p, q \leq \infty, 1 / p+1 / q=1$. Let $\left|f^{\prime \prime \prime}\right|^{p}:[a, b] \rightarrow \mathbb{R}$ be an R-integrable function. Then for $1<p \leq \infty$ we 
have

$$
\begin{aligned}
& \mid \int_{a}^{b} f(t) g(t) d t-\int_{b-\lambda}^{b} f(t) d t-f^{\prime}(b-\lambda)\left(\frac{\lambda^{2}}{2}-b \lambda+\int_{a}^{b} x g(x) d x\right) \\
& +\frac{f^{\prime \prime}(b)}{2}\left(\frac{\lambda^{3}}{3}-\int_{a}^{b} g(x)(x-b+\lambda)^{2} d x\right) \mid \leq\left\|f^{\prime \prime \prime}\right\|_{p}\left(\int_{a}^{b-\lambda} \mid \frac{1}{3} \int_{a}^{s} x^{3} g(x) d x\right. \\
& -s \int_{a}^{s} x^{2} g(x) d x-\left[(b-\lambda)^{2}-2 s(b-\lambda)\right] \int_{a}^{b} x g(x) d x-s^{2} \int_{s}^{b} x g(x) d x-\frac{s^{3}}{3} \int_{a}^{s} g(x) d x \\
& -\left.\frac{(b-\lambda-s)^{2}}{2}\left(\frac{\lambda^{2}}{2}-\lambda b\right)\right|^{q}+\int_{b-\lambda}^{b} \mid \frac{1}{3} \int_{a}^{s} x^{3} g(x) d x-s \int_{a}^{s} x^{2} g(x) d x \\
& -\left[(b-\lambda)^{2}-2 s(b-\lambda)\right] \int_{a}^{s} x g(x) d x-s^{2} \int_{s}^{b} x g(x) d x+\frac{s^{3}}{3} \int_{s}^{b} g(x) d x \\
& \left.+\frac{(s-b+\lambda)^{4}}{12}+\frac{(s-b+\lambda)^{3} \lambda}{2}\left[\frac{\lambda}{3}+\frac{2}{3}(b-s)\right]+\frac{2}{3} \lambda(b-\lambda)^{3}-\left.s \lambda(b-\lambda)^{2}\right|^{q} d t\right) .
\end{aligned}
$$

and the constant on the right-hand side of (3.6) is sharp, while for $p=1$ we have

$$
\begin{aligned}
& \mid \int_{a}^{b} f(t) g(t) d t-\int_{b-\lambda}^{b} f(t) d t-f^{\prime}(b-\lambda)\left(\frac{\lambda^{2}}{2}-b \lambda+\int_{a}^{b} x g(x) d x\right) \\
& +\frac{f^{\prime \prime}(b)}{2}\left(\frac{\lambda^{3}}{3}-\int_{a}^{b} g(x)(x-b+\lambda)^{2} d x\right) \mid \leq\left\|f^{\prime \prime \prime}\right\|_{1} \max \left\{M_{1}, M_{2}\right\}
\end{aligned}
$$

where

$$
\begin{aligned}
& M_{1}=\max _{s \in[a, b-\lambda]}\{\left\{\frac{1}{3} \int_{a}^{s} x^{3} g(x) d x-s \int_{a}^{s} x^{2} g(x) d x-\left[(b-\lambda)^{2}-2 s(b-\lambda)\right] \int_{a}^{b} x g(x) d x\right. \\
&\left.-s^{2} \int_{s}^{b} x g(x) d x-\frac{s^{3}}{3} \int_{a}^{s} g(x) d x-\frac{(b-\lambda-s)^{2}}{2}\left(\frac{\lambda^{2}}{2}-\lambda b\right)\right\}, \\
& M_{2}=\max _{s \in[b-\lambda, b]}\left\{\frac{1}{3} \int_{a}^{s} x^{3} g(x) d x-s \int_{a}^{s} x^{2} g(x) d x\right. \\
&-\left[(b-\lambda)^{2}-2 s(b-\lambda)\right] \int_{a}^{s} x g(x) d x-s^{2} \int_{s}^{b} x g(x) d x+\frac{s^{3}}{3} \int_{s}^{b} g(x) d x \\
&+\frac{(s-b+\lambda)^{4}}{12}+\frac{(s-b+\lambda)^{3} \lambda}{2}\left[\frac{\lambda}{3}+\frac{2}{3}(b-s)\right] \\
&\left.+\frac{2}{3} \lambda(b-\lambda)^{3}-s \lambda(b-\lambda)^{2}\right\} .
\end{aligned}
$$

and the constant on the right-hand side of (3.7) is the best possible. 


\section{Generalizations related to the bounds for the Čebyšev functional}

Let $f, h:[a, b] \rightarrow \mathbb{R}$ be Lebesgue integrable functions. We define the Čebyšev functional $T(f, h)$ by

$$
T(f, h):=\frac{1}{b-a} \int_{a}^{b} f(t) h(t) d t-\frac{1}{b-a} \int_{a}^{b} f(t) d t \cdot \frac{1}{b-a} \int_{a}^{b} h(t) d t .
$$

In [2] Cerone and Dragomir proved the following theorems:

Theorem 4.1. Let $f:[a, b] \rightarrow \mathbb{R}$ be a Lebesgue integrable function and $h:$ $[a, b] \rightarrow \mathbb{R}$ be an absolutely continuous function with $(\cdot-a)(b-\cdot)\left[h^{\prime}\right]^{2} \in L_{1}[a, b]$. Then we have the inequality

$$
|T(f, h)| \leq \frac{1}{\sqrt{2}}[T(f, f)]^{\frac{1}{2}} \frac{1}{\sqrt{b-a}}\left(\int_{a}^{b}(x-a)(b-x)\left[h^{\prime}(x)\right]^{2} d x\right)^{\frac{1}{2}} .
$$

The constant $\frac{1}{\sqrt{2}}$ in (4.1) is the best possible.

Theorem 4.2. Assume that $h:[a, b] \rightarrow \mathbb{R}$ is monotonic nondecreasing on $[a, b]$ and $f:[a, b] \rightarrow \mathbb{R}$ is absolutely continuous with $f^{\prime} \in L_{\infty}[a, b]$. Then we have the inequality

$$
|T(f, h)| \leq \frac{1}{2(b-a)}\left\|f^{\prime}\right\|_{\infty} \int_{a}^{b}(x-a)(b-x) d h(x) .
$$

The constant $\frac{1}{2}$ in (4.2) is the best possible.

In the sequel we use the above theorems to obtain some new bounds for integrals on the left hand side in the perturbed version of identities (2.2) and (2.6).

Firstly, let us denote

$$
\Omega_{i}(s)=\int_{a}^{b} G_{i}(x) g_{A G 2}(x, s) d x, \quad i=1,2 .
$$

Theorem 4.3. Let $f \in C^{n+1}[a, b]$ for some $n \geq 3$ with $(\cdot-a)(b-\cdot)\left[f^{(n+1)}\right]^{2} \in$ $L_{1}[a, b]$. Let $g, p:[a, b] \rightarrow \mathbb{R}$ be integrable functions such that $p$ is positive and $0 \leq g \leq 1$. Let $\int_{a}^{a+\lambda} p(t) d t=\int_{a}^{b} g(t) p(t) d t$ and let functions $G_{1}$ and $\Omega_{1}$ be 
defined by (2.1) and (4.3). Then

$$
\begin{aligned}
& \int_{a}^{a+\lambda} f(t) p(t) d t-\int_{a}^{b} f(t) g(t) p(t) d t+\sum_{i=0}^{\alpha} \frac{f^{(i+1)}(a)}{i !} \int_{a}^{b} G_{1}(x)(x-a)^{i} d x \\
& +\sum_{j=0}^{n-\alpha-3} f^{(\alpha+j+2)}(a+\lambda)\left[\sum_{i=0}^{j} \frac{(-\lambda)^{j-i}}{(\alpha+1+i) !(j-i) !}\left(\int_{a}^{b}(x-a)^{\alpha+1+i} G_{1}(x) d x\right)\right] \\
& +\frac{f^{(n-1)}(b)-f^{(n-1)}(a)}{b-a} \int_{a}^{b} \Omega_{1}(s) d s=S_{n}^{1}(f ; a, b)
\end{aligned}
$$

where the remainder $S_{n}^{1}(f ; a, b)$ satisfies the estimation

$$
\left|S_{n}^{1}(f ; a, b)\right| \leq \frac{\sqrt{b-a}}{\sqrt{2}}\left[T\left(\Omega_{1}, \Omega_{1}\right)\right]^{\frac{1}{2}}\left|\int_{a}^{b}(s-a)(b-s)\left[f^{(n+1)}(s)\right]^{2} d s\right|^{\frac{1}{2}} .
$$

Proof. Applying Theorem 4.1 for $f \rightarrow \Omega_{1}$ and $h \rightarrow f^{(n)}$ we obtain

$$
\begin{aligned}
& \left|\frac{1}{b-a} \int_{a}^{b} \Omega_{1}(s) f^{(n)}(s) d s-\frac{1}{b-a} \int_{a}^{b} \Omega_{1}(s) d s \cdot \frac{1}{b-a} \int_{a}^{b} f^{(n)}(s) d s\right| \\
& \leq \frac{1}{\sqrt{2}}\left[T\left(\Omega_{1}, \Omega_{1}\right)\right]^{\frac{1}{2}} \frac{1}{\sqrt{b-a}}\left|\int_{a}^{b}(s-a)(b-s)\left[f^{(n+1)}(s)\right]^{2} d t\right|^{\frac{1}{2}} .
\end{aligned}
$$

Now if we add

$$
\frac{1}{b-a} \int_{a}^{b} \Omega_{1}(s) d s \int_{a}^{b} f^{(n)}(s) d s=\frac{f^{(n-1)}(b)-f^{(n-1)}(a)}{b-a} \int_{a}^{b} \Omega_{1}(s) d s
$$

to both sides of identity (2.2) and use inequality (4.6) we obtain representation (4.4) and bound (4.5).

Similarly, using identity (2.6) we obtain the following result:

Theorem 4.4. Let $f \in C^{n+1}[a, b]$ for some $n \geq 3$ with $(\cdot-a)(b-\cdot)\left[f^{(n+1)}\right]^{2} \in$ $L_{1}[a, b]$. Let $g, p:[a, b] \rightarrow \mathbb{R}$ be integrable functions such that $p$ is positive and $0 \leq g \leq 1$. Let $\int_{b-\lambda}^{b} p(t) d t=\int_{a}^{b} g(t) p(t) d t$ and let functions $G_{2}$ and $\Omega_{2}$ be 
defined by (2.5) and (4.3). Then

$$
\begin{aligned}
& \int_{a}^{b} f(t) g(t) p(t) d t-\int_{b-\lambda}^{b} f(t) p(t) d t+\sum_{i=0}^{\alpha} \frac{f^{(i+1)}(b-\lambda)}{i !} \int_{a}^{b} G_{2}(x)(x-b+\lambda)^{i} d x \\
& +\sum_{j=0}^{n-\alpha-3} f^{(\alpha+j+2)}(b)\left(\sum_{i=0}^{j} \frac{(-\lambda)^{j-i}}{(\alpha+1+i) !(j-i) !}\left(\int_{a}^{b}(x-b+\lambda)^{\alpha+1+i} G_{2}(x) d x\right)\right) \\
& +\frac{f^{(n-1)}(b)-f^{(n-1)}(a)}{b-a} \int_{a}^{b} \Omega_{2}(s) d s=S_{n}^{2}(f ; a, b),
\end{aligned}
$$

where the remainder $S_{n}^{2}(f ; a, b)$ satisfies the estimation

$$
\left|S_{n}^{2}(f ; a, b)\right| \leq \frac{\sqrt{b-a}}{\sqrt{2}}\left[T\left(\Omega_{2}, \Omega_{2}\right)\right]^{\frac{1}{2}}\left|\int_{a}^{b}(s-a)(b-s)\left[f^{(n+1)}(s)\right]^{2} d s\right|^{\frac{1}{2}} .
$$

Proof. Similar to the proof of Theorem 4.3.

The following Grüss-type inequalities also hold.

Theorem 4.5. Let $f \in C^{n+1}[a, b]$ for some $n \geq 3$ and $f^{(n+1)} \geq 0$ on $[a, b]$. Let functions $\Omega_{i}, i=1,2$ be defined by (4.3).

(a) Let $\int_{a}^{a+\lambda} p(t) d t=\int_{a}^{b} g(t) p(t) d t$. Then we have representation (4.4) and the remainder $S_{n}^{1}(f ; a, b)$ satisfies the bound

$\left|S_{n}^{1}(f ; a, b)\right| \leq(b-a)\left\|\Omega_{1}^{\prime}\right\|_{\infty}\left\{\frac{f^{(n-1)}(b)+f^{(n-1)}(a)}{2}-\frac{f^{(n-2)}(b)-f^{(n-2)}(a)}{b-a}\right\}$.

(b) Let $\int_{b-\lambda}^{b} p(t) d t=\int_{a}^{b} g(t) p(t) d t$. Then we have representation (4.7) and the remainder $S_{n}^{2}(f ; a, b)$ satisfies the bound

$\left|S_{n}^{2}(f ; a, b)\right| \leq(b-a)\left\|\Omega_{2}^{\prime}\right\|_{\infty}\left\{\frac{f^{(n-1)}(b)+f^{(n-1)}(a)}{2}-\frac{f^{(n-2)}(b)-f^{(n-2)}(a)}{b-a}\right\}$.

Proof. (a) Applying Theorem 4.2 for $f \rightarrow \Omega_{1}, h \rightarrow f^{(n)}$ and multiplying by $(b-a)$ we obtain

$$
\begin{aligned}
& \left|\int_{a}^{b} \Omega_{1}(s) f^{(n)}(s) d s-\int_{a}^{b} \Omega_{1}(s) d s \cdot \frac{1}{b-a} \int_{a}^{b} f^{(n)}(s) d s\right| \\
& \leq \frac{1}{2}\left\|\Omega_{1}^{\prime}\right\|_{\infty} \int_{a}^{b}(s-a)(b-s) f^{(n+1)}(s) d s .
\end{aligned}
$$


Since

$$
\begin{aligned}
& \int_{a}^{b}(s-a)(b-s) f^{(n+1)}(s) d s=\int_{a}^{b}[2 s-(a+b)] f^{(n)}(s) d s \\
& =(b-a)\left[f^{(n-1)}(b)+f^{(n-1)}(a)\right]-2\left(f^{(n-2)}(b)-f^{(n-2)}(a)\right) .
\end{aligned}
$$

Using representation (2.2) and inequality (4.9) we deduce (4.8).

(b) Similar to the (a)-part.

Acknowledgements. The research of the first author was supported by the Ministry of Education and Science of the Russian Federation (the Agreement number No. 02a03.21.0008).

\section{References}

[1] R.P. Agarwal, P.J.Y. Wong, Error Inequalities in Polynomial Interpolation and Their Applications, Mathematics and its Applications 262, Kluwer Academic Publishers, Dordrecht, 1993.

[2] P. Cerone, S.S. Dragomir, Some new bounds for the Čebyšev functional in terms of the first derivative and applications, J. Math. Inequal. 8(1) (2014), 159-170.

[3] P.J. Davis, Interpolation and Approximation, Blaisdell Publishing Co. Ginn and Co., New York-Toronto-London, 1963.

[4] V.L. Gontscharoff, Theory of interpolation and approximation of functions, Gostekhizdat, Moscow, 1954.

[5] J. Jakšetić, J. Pečarić, Steffensen's inequality for positive measures, Math. Inequal. Appl. 18(3) (2015), 1159-1170.

[6] D.S. Mitrinović, The Steffensen inequality, Univ. Beograd. Publ. Elektrotehn. Fak. Ser. Mat. Fiz. 247-273 (1969), 1-14.

[7] J. Pečarić, A. Perušić, K. Smoljak, Generalizations of Steffensen's inequality by Abel-Gontscharoff polynomial, Khayyam Journal of Mathematics, 1(1) (2015), 45-61.

[8] J.E. Pečarić, F. Proschan, Y.L. Tong, Convex functions, partial orderings, and statistical applications, Mathematics in science and engineering 187, Academic Press, 1992. 
[9] J. Pečarić, K. Smoljak Kalamir, S. Varošanec, Steffensen's and related inequalities (A comprehensive survey and recent advances), Monograhps in inequalities 7, Element, Zagreb, 2014.

[10] J.F. Steffensen, On certain inequalities between mean values and their application to actuarial problems, Skand. Aktuarietids. (1918), 82-97.

[11] J.M. Whittaker, Interpolatory Function Theory, Cambridge University Press, London, 1935.

Josip Pečarić,

Faculty of Textile Technology,

University of Zagreb,

Prilaz baruna Filipovića 28a, 10000 Zagreb, Croatia

and RUDN University,

Miklukho-Maklaya str. 6, 117198 Moscow, Russia

Email: pecaric@element.hr

Anamarija Perušić Pribanić,

Faculty of Civil Engineering,

University of Rijeka,

Radmile Matejčić 3, 51000 Rijeka, Croatia

Email: anamarija.perusic@gradri.uniri.hr

Ksenija Smoljak Kalamir,

Faculty of Textile Technology,

University of Zagreb,

Prilaz baruna Filipovića 28a, 10000 Zagreb, Croatia

Email: ksmoljak@ttf.hr 